\title{
Evaluation of Double Loop IC Module for Inductive Coupled Fed RFID Tag Wire Embedded Antenna
}

\author{
N. A. Murad ${ }^{1,2}$, M. H. M Salleh ${ }^{2}$, N. Q. Azmi ${ }^{1}$, A. R. Tanvir ${ }^{1}$ and A. A. Z. A. Nizam ${ }^{1}$ \\ ${ }^{1}$ Advanced RF and Microwave Research Group (ARFMRG), School of Electrical Engineering, Faculty of Engineering, \\ Universiti Teknologi Malaysia (UTM), 81310, UTMJB, Johor, Malaysia. \\ ${ }^{2}$ HID GLOBAL Sdn. Bhd. Bandar Indahpura, 81000 Kulai, Johor
}

\begin{tabular}{l} 
Article Info \\
\hline Article history: \\
Received May 2, 202 \\
Revised Sep 14, 2020 \\
Accepted Oct 1, 2020 \\
\hline Keyword: \\
RFID tag \\
Inductive coupled \\
Dipole antenna \\
CST \\
Wire embedded
\end{tabular}

\begin{abstract}
This paper presents an evaluation on double loop integrated circuit module for inductive couple fed RFID Tag. The inductive couple feed gives advantage especially for flexible wire embedded antenna in controlling the manufacturing varians as the chip is not directly connected to the antenna. Thus electrostatic discharged effect and manufacturing errors can be minimized. An inductor loop is directly connected to the RFID chip before it is placed to indirectly coupled the energy from a meandered dipole antenna. The coupled energy should be enough to turn on the chip to communicate with the reader. The inductive coupled energy is achieved by manipulating the near field magnetic field between the antenna body and the inductor loop. To evaluate the performance, the antenna and the inductive feeding loop is designed to operate at RFID UHF band $(860 \mathrm{MHz}-960 \mathrm{MHz})$ and simulated using CST software. The antenna body impedance is evaluated to match the impedance of the chip and the loop. It is confirmed that the double loop inductor has higher inductance values and thus should be counted in conjugate impedance between the antenna body and the chip module. The tag with overall dimension of $60 \mathrm{~mm} \times 16 \mathrm{~mm}$ can be read at distance at least 9 meters through out the band.
\end{abstract}

Copyright $(2) 2020$ Institute of Advanced Engineering and Science. All rights reserved.

\section{Corresponding Author:}

N. A. Murad,

Faculty of Engineering,

Universiti Teknologi Malaysia (UTM),

81310, UTMJB, Johor, Malaysia.,

Email : noorasniza@utm.my

\section{INTRODUCTION}

Radio Frequency Identification (RFID) is a wireless communication system that enable contactless data transfer. Two main components in RFID system are reader and tag. An RFID reader would read data or information on RFID tag by means of electromagnetic coupling or radio waves. Reader broadcast queries for information and tags within the reader transmission range would response with required information. The types of RFID commonly identified based on the operational radio waves spectrum. There are low frequency (LF), high frequency (HF) and ultra high frequency (UHF). LF (125-134 kHz) and HF (13.56 MHz) normally used for short range applications while UHF (433 MHz, 860-960 MHz) for longer range [1]. RFID operating in the UHF band gaining interests in many applications such as for inventory, supply-chain management, electronic tolling system and many more where long read distance is required. Dipole antenna is commonly used in UHF RFID tags due to its simplicity and omnidirectional properties. An RFID chip can be connected to the two arms of the dipole which will be turned on for data transfer once enough energy is captured by the dipole and transferred to the feed point. A good conjugate matching between the antenna impedance, $Z_{a}$ and the chip impedance, $Z_{c}$ is a must to have efficient energy transfer. There are numbers of matching techniques reported such as $\mathrm{T}$-shape and inductive coupling [2,3]. 
Designed tag is subject to process variation and failures during mass production manufacturing. A large size dipole antenna connected to the chip will induce mechanical stress on the bonding which could break the connection especially on flexible tags. A direct connection of the antenna to the chip will transfer the energy of any electrostatic discharge directly to the chip which could destroy the functionalities of the chip. Thus, a chip module is introduced where the bare chip is bonded to a loop and packaged to protect the chip itself during the production and its life cycle. The loop in the chip module act as a couple loops that can inductively gain energy from the dipole antenna. Wire embedded antenna is one of commonly used antenna in RFID tags. The wire is embedded on a substrate using ultrasonic sonotrode at certain frequency of vibration. During the process, deformation may occure due to the pressure between the wire and the substrate. Optimization to reduce the deformation is necessary. The optimization needs to be tolerable with the feeding element that connect the antenna to the chip. Therefore, this paper reports the evaluation on the UHF RFID wire embedded antenna with inductively couple feed. The combination of both technologies could enhance the product robustness, durability and reliability.

\section{RESEARCH METHOD}

The structure design is divided into two, i) the inductor loop, and ii) the tag antenna. The inductor loop is studied in term of the inductance value at different dimensions. Once the impedance of the loop is known, it is connected to the RFID chip. The antenna then is designed to have conjugate impedance of the loop and the chip for matching purposes at the required frequency.

\subsection{Inductor Loop}

An inductor is basically a passive electrical component which normally built in the form of coil wire. Inductor takes the advantage of the relationship between magnetism and electricity where induced current can be produced with certain condition on magnetic field. The loop inductance calculation can be found in [9-10] with different shapes. An inductance, $L$ of a square inductor loop (Figure 1 ) can be calculated by [9]:

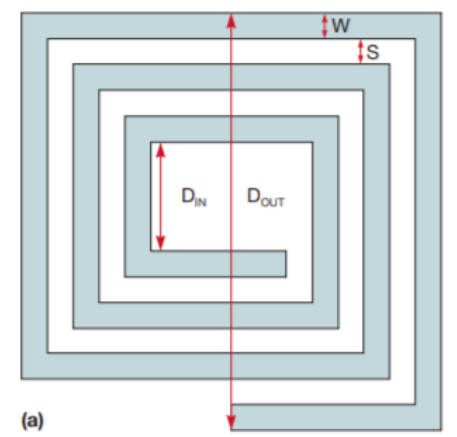

Figure 1. Square inductor loop [9]

$$
L=\left[\frac{\mu_{0} N^{2} D_{\text {avg }} C_{1}}{2}\right]\left[\ln \left(\frac{C_{2}}{\rho}\right)+C_{3} \rho+C_{4} \rho^{2}\right]
$$

Where $N$ is the number of turns, $\mu_{0}$ is the vacuum permeability, $4 \pi \times 10-7 ; \rho$ is the fill ratio, $\left(D_{\text {OUT }}-D_{I N}\right) /\left(D_{\text {OUT }}+D_{I N}\right) ; D_{A V G}$ is the average diameter, $\left(D_{I N}+D_{\text {OUT }}\right) / 2 ;$ and $\mathrm{C}_{1}=1.27, \mathrm{C}_{2}=2.07, \mathrm{C}_{3}=0.18$, and $\mathrm{C}_{4}=0.13$. Based on the equation, theoretically the inductance value is physically depending on the fill ratio and average diameter of the loop.

\subsection{Meandered Dipole Tag Antenna}

Dipole antenna is a widely used in RFID tags due to its simplicity and omnidirectional pattern [4-8]. Dipole antenna is basically designed with two arms wire at half wavelength in total length at the target frequency. Considering free space environment, a dipole antenna length is $166 \mathrm{~mm}$ at $900 \mathrm{MHz}$. Smaller dimension is preferable for most RFID applications. Meandering is one of common methods used in reducing the dipole antenna dimension. Meandering is a process of bending the arms wire and it can be repeated while maintaining the overall length of the wire. However, the bending degrades the electrical properties of the antenna with the current lines flowing in opposite directions through adjacent paths and increases the radiation resistance. The RFID chip can be directly connected at the centre of the dipole, but a matching 
circuit is needed to ensure efficient capture of the RF signal. Different matching techniques is reported in $[2,3]$. Inductive coupled method is used in this work.

Inductive coupled feed for RFID antenna design can be found in [3]. The paper reported analysis of a single inductive coupled loop. This work proposed two loops to be packaged in a chip module. The basic theory presented in [3] is applied in this work. Referring to [3] the input impedance of the antenna is given as:

$$
Z_{a}=R_{a}+j X_{a}=Z_{l o o p}+\frac{(2 \pi f M)^{2}}{Z_{r b}}
$$

Where $Z_{\text {loop }}$ and $Z_{r b}$ are individual impedances of the feed loop and radiating body respectively. $M$ is the mutual inductance between the radiating body and feeding loop. Thus, the strength of the coupling is controlled by the distance between the loop and the radiating body as well as the shape of the loop [3]. It was also reported that at resonance, $R_{a}$ depends only on $M$ while $X_{a}$ depends on the loop inductance, $L_{\text {loop }}$. Therefore, two components of the antenna impedance can be adjusted independently to match the arbitrary impedance of the chip, $Z_{c}=R_{c}+j X_{c}$.

The proposed structure is shown in Figure 2. The meandered antenna body is designed using 110 microns' wire on $0.2 \mathrm{~mm}$ polycarbonate with dielectric constant of 2.9. A double loop inductor is connected directly to the chip as a module. The chip is connected at the feed terminal of the loop which would be turned on when enough energy is coupled from the meandered dipole.

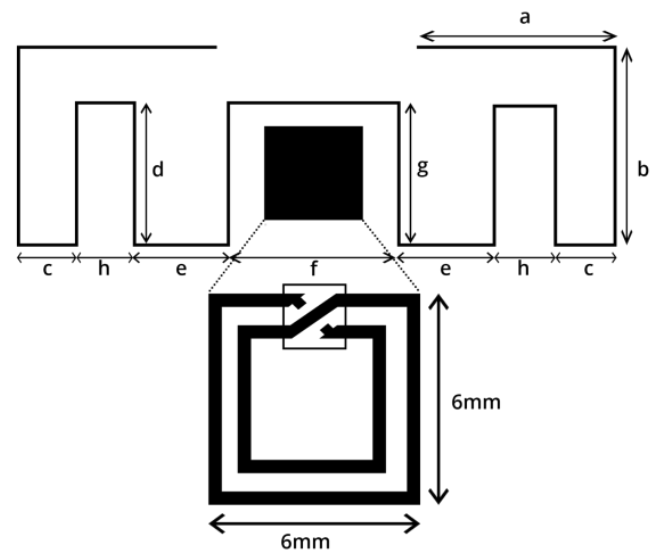

Figure 2. The antenna configuration

In this work, the Impinj Monza 5 chip is used with intrinsic capacitance of $0.825 \mathrm{pF}$; typical mount parasitic capacitance of $0.245 \mathrm{pF}$ and the resistance of $1.2 \mathrm{k} \Omega$. The typical capacitance and resistance parallel circuit of the chip is simulated. The response curve shows the impedance of the chip is $Z_{c}=13-\mathrm{j} 153$ at $915 \mathrm{MHz}$.

The loop is a planar double square loop with outer dimension $6 \mathrm{~mm} \times 6 \mathrm{~mm}$. The trace line width is $0.5 \mathrm{~mm}$ and the gap between the trace is also made $0.5 \mathrm{~mm}$. The calculated inductance of the loop based on equation (2) is $0.22 \mathrm{nH}$ which give $Z_{\text {loop }}=\mathrm{j} 131 \Omega$. The chip module then is placed to feed the meandered dipole. The antenna dimensions are tabulated in Table 1.

Table 1. The antenna dimensions.

\begin{tabular}{cc}
\hline Variable & Dimension $(\mathrm{mm})$ \\
\hline $\mathrm{a}$ & 22 \\
$\mathrm{~b}$ & 16 \\
$\mathrm{c}$ & 3 \\
$\mathrm{~d}$ & 14 \\
$\mathrm{e}$ & 18 \\
$\mathrm{~g}$ & 13 \\
$\mathrm{~h}$ & 3 \\
\hline
\end{tabular}

\section{RESULTS AND ANALYSIS}

3.1. Inductor Loop 
Inductance of the square inductor loops with different physical parameters are studied. The structures have been simulated using CST software. The study starts with a single $0.2 \mathrm{~mm}$ copper line square loop on $0.3 \mathrm{~mm}$ FR4 board. Figure 3 shows that by increasing the outer dimension of the square, $D_{\text {out }}$, the reactance value is increasing, showing that the inductance value is increased. The same pattern is observed from double loops $0.2 \mathrm{~mm}$ copper line with $0.1 \mathrm{~mm}$ gap between the lines as shown in Figure 3 . But higher reactance is observed in double loops compared to single loop showing higher inductance in double loops.

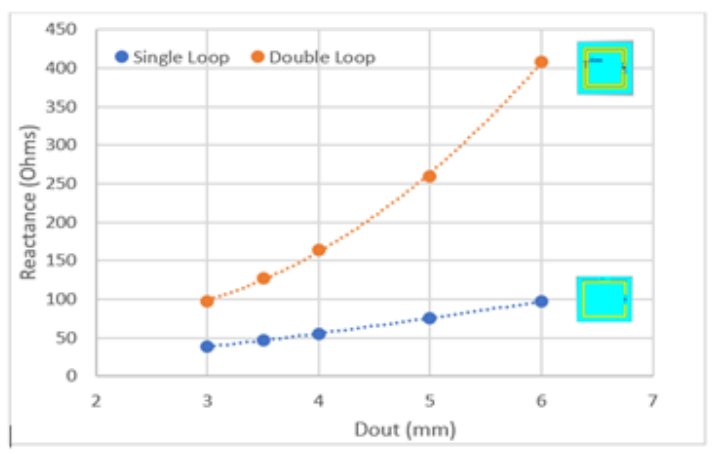

Figure 3 Single and double loop reactance.

\subsection{Meandered dipole antenna}

The designed antenna has been simulated using Computer Simulation Technology (CST) software. Figure 4 (a) and (b) shows the resistance components, $R a$ and the reactance component, $X a$ against the frequencies when the antenna length of section $a$ is varying. It shows that the longer the length, the maximum resistance responses shift to the lower band where the maximum $R_{a}$ is occurred at the antenna self resonance, $f_{0}$. The trend is also seen in the reactance responses. However, the antenna reactance value is much less compared to the arbitrary chip impedance at shorter length

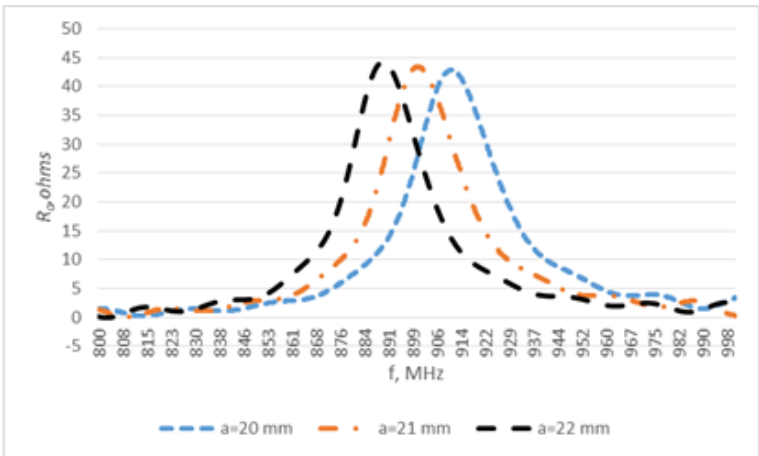

(a)

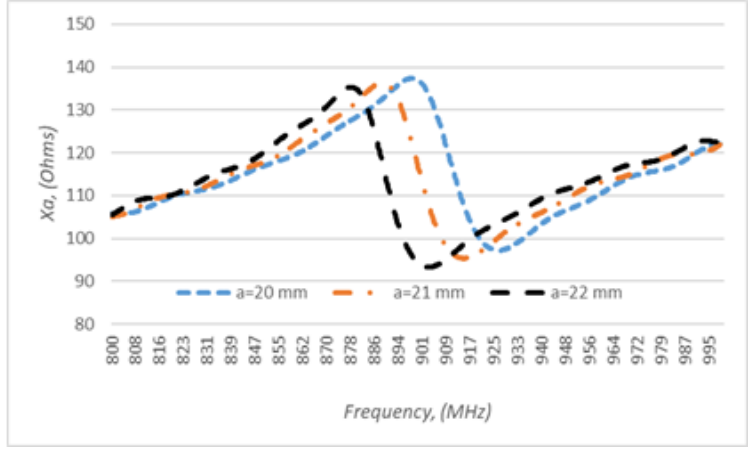

(b)

Figure 4. The antenna (a) resistance against frequencies and (b) reactance against frequencies. 


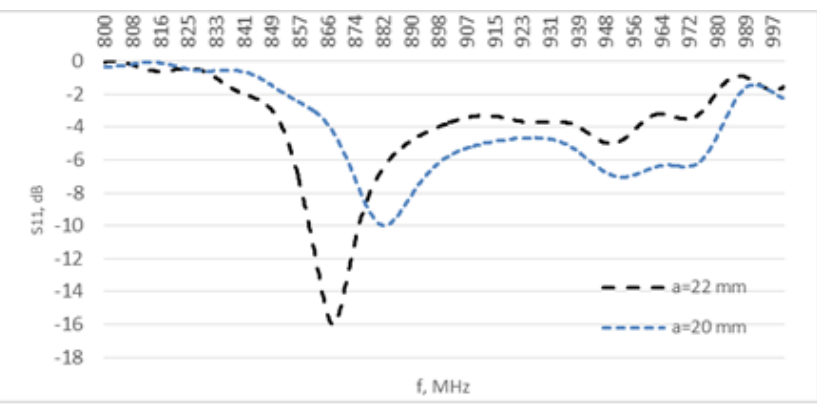

Figure 5. The reflection coefficient against frequencies.

Figure 5 shows the reflection coefficient response with $\mathrm{a}=22 \mathrm{~mm}$ and $\mathrm{a}=20 \mathrm{~mm}$. It shows that the reflection at resonance is better with $a=22 \mathrm{~mm}$ with response to the conjugate reactance in Figure 4 (b). The radiation pattern of the antenna with $\mathrm{a}=22 \mathrm{~mm}$ is shown in Figure 6 with maximum gain of $1.37 \mathrm{dBi}$ at 915 $\mathrm{MHz}$. The maximum gain over the frequencies is used to plot the tag read range.

The Friis equation is applied to the post processing steps in CST to determine the read range. Friis equation is given by [6]:

$$
P_{r}=\operatorname{EIRP}\left(G_{r}\right)\left(\frac{\lambda}{4 \pi d}\right)^{2}
$$

Where $P_{r}$ is the power received, EIRP is the effective isotropic radiated power which is $4 \mathrm{~W}$ for FCC standard, $G_{r}$ is the tag antenna gain, and $d$ is the read distance. The meandered dipole with a double loop IC module $\left(D_{\text {OUT }}=5 \mathrm{~mm}\right)$ have been fabricated and measured. The read distance is plotted against frequencies in Figure 7. It shows that the tag can be read longer than 9 meters from $860 \mathrm{MHz}$ to $960 \mathrm{MHz}$ to cover overall UHF RFID band.

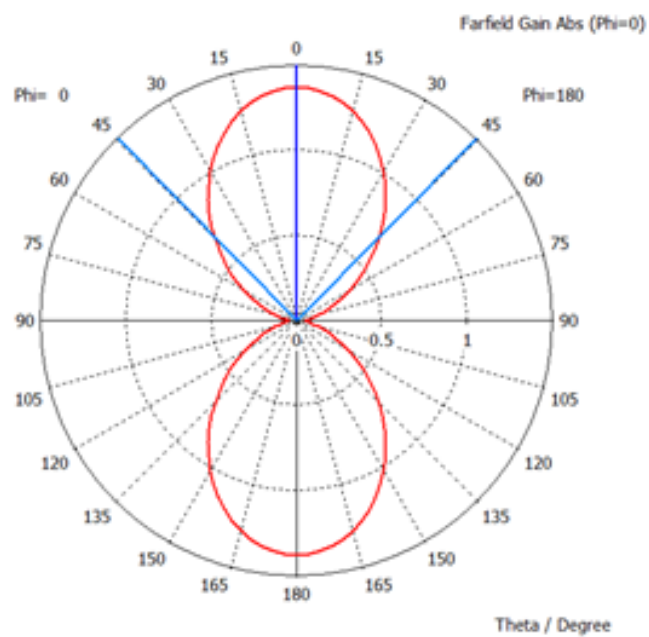

Figure 6 . The radiation pattern

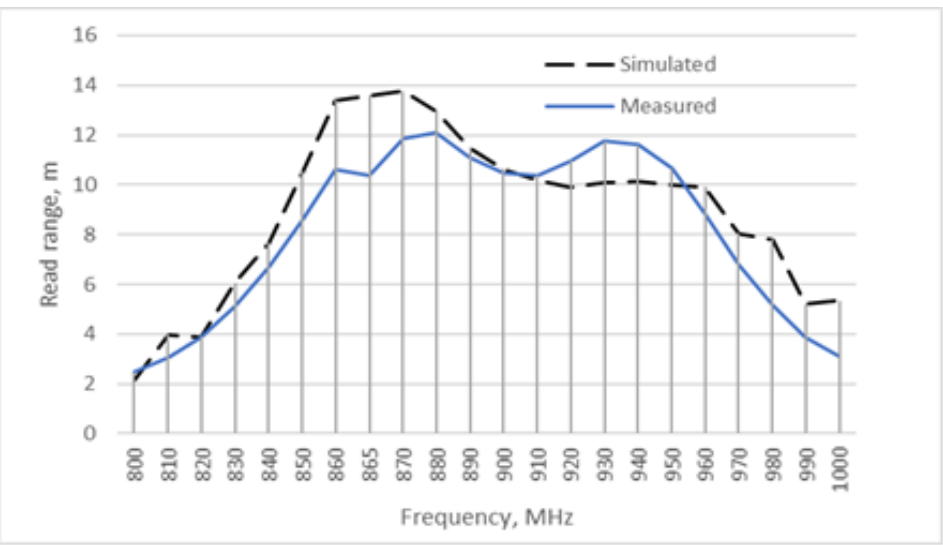

Figure 7. The read range against frequencies 


\section{CONCLUSION}

A double loop IC module is evaluated to fed a wire embedded meandered dipole antenna for UHF RFID tag. A proper matching should be considered to ensure optimum couple energy to turn on the chip. Inductive couple feed give advantage in enhancing the tag bandwidth as well as minimizing the manufacturing rejection rate and failures. In this work, a good bandwith with read range greater than 9 meters is observed over the UHF RFID band.

\section{ACKNOWLEDGEMENTS}

The authors would like to acknowledge the support from HID GLOBAL Sdn. Bhd., Advance RF and Microwave Research Group, School of Elctrical Engineering, and Universiti Teknologi Malaysia under grant number $21 \mathrm{H} 69$.

\section{REFERENCES}

[1] Daniel M. Dobkin, " The RF in RFID: UHF RFID in Practice," $2^{\text {nd }}$ Ed., Elsevier, 2013.

[2] Gaetano Marrocco, " The Art of UHF RFID Antenna Design: Impedance-Matching and Size-Reduction Techniques," IEEE Antennas and Propagation Magazine, vol. 50, pp. 66-79, 2008.

[3] H.-W. Son and C.-S. Pyo, "Design of RFID tag antennas using an inductively coupled feed," Electronics Letters, Vols 41, No.18, 2005.

[4] Jibish Mathew Manju Abraham C.K. Aanandan Thomaskutty Mathew "A broadband miniaturized UHF RFID dipole tag antenna with tapered meandered arms," Microwave and Optical Technology Letter, Vol. 59, No. 5, 2017.

[5] S. Das, H. Saghlatoon, P. Mousavi and A. K. Iyer, "A Highly Miniaturized and Inherently Conjugately Matched Folded Dipole-Based RFID Tag Antenna," in IEEE Access, vol. 7, pp. 101658-101664, 2019.

[6] I. Aznabet, M. A. Ennasar, O. El Mrabet, G. Andia-Vera, M. Khalladi, and S. Tedjni, "A Broadband Modified T-Shaped Planar Dipole Antenna for UHF RFID Tag Applications," Progress In Electromagnetics Research C, Vol. 73, 137-144, 2017.

[7] P. Soboll, V. Wienstroer and R. Kronberger, "Innovating RFID for Future Applications: A Capacitive Coupled Antenna Design for UHF RFID Application," in IEEE Microwave Magazine, vol. 17, no. 2, pp. 65-69, Feb. 2016.

[8] Xiuwei Xuan, Lianrong Lv, and Kun Li, “A Miniaturized Meandered Dipole UHF RFID Tag Antenna for Flexible Application," International Journal of Antennas and Propagation, vol. 2016, Article ID 2951659, 7 pages, 2016.

[9] Zhao, Jonsenser "A new calculation for designing multilayer planar spiral inductors." Edn -Boston then Denver then Highlands Ranch Co-. 55. 37-40, 2010.

[10] Michal Ulvr "Design of PCB search coils for AC magnetic flux density measurement." AIP Advances 8, 047505. 2018.

[11] D.M.Pozar, "Microwave Engineering." $4^{\text {th }}$ Ed., Wiley, 2011. 